\title{
Preparation of Cucurbituril Anchored Silica Gel by Cross Polymerization and Its Chromatographic Applications
}

\author{
Won Jo Cheong," Joung Ho Go, Yoon Suk Baik, Sung Soon Kim, Erumaipatty R. Nagarajan," \\ Narayanan Selvapalam, ${ }^{*}$ Young Ho Ko, ${ }^{*}$ and Kimoon Kim ${ }^{\dagger+*}$ \\ Department of Chemistry and Fine Nano Center, Inha University; Incheon 402-751, Korea. "E-mail: wjcheong@inha.ac.kr \\ ${ }^{\dagger}$ National Creative Research Initative Center for Smart Supramolecules, and Department of Chemistry; Pohang University \\ of Science and Techology (POSTECH), Pohang 790-784, Korea. "E-mail. kkim(apostech ac.kr
}

Received April 29, 2008

\begin{abstract}
A new chromatographic stationary phase has been prepared by cross polymerization between allylsilica and perallyloxycucurbit[6]uril and characterized by elemental analysis and FT-IR spectroscopy. The double endcapping has been proven to improve the separation efficiency of the cucurbituril-based stationary phase material. The first end-capping was cartied out when allylsilica was made. The second end-capping was done as the final step of the whole process, and the use of a mixture of hexametlyyldisilazane (HMDS) and trimethylchlorosilane (TMCS) as an end-capping reagent was found better than the use of only HMDS or TMCS. Our stationary phase has shown generally good results in separation of nonpolar and polar analytes. This phase showed even better separation perfornance than the commercial C18 phase for the case where hostguest chemistry was properly incorporated in solute retention.
\end{abstract}

Key Words : Perallyloxycucurbituril, Allyl silica, Cross polymerization, Double end-capping, Stationary phase

\section{Introduction}

The host family cucurbit[n]uril ( $\mathrm{CB}[n], n=5-10)$, comprising $n$ glycoluril units, has a hydrophobic cavity and two identical carbonyl-laced portals, ${ }^{1-\%}$ which allow them to form stable host-guest complexes with various guest molecules and ions. Supramolecular chemistry of $\mathrm{CB}[n]$, including their host-guest chemistry and applications has been studied extensively and summarized in two topical review articles. ${ }^{5,6}$ Although the $\mathrm{CB}[n]$ are potentially as useful as well known host molecules such as calixarenes and cyclodextrins, their practical applications including their use in separation science have been limited mainly due to the difficulty in introducing functional groups on their surfaces. No method for direct fuctionalization of $\mathrm{CB}[n]$ was available until recently, which may be attributed to the high chemical stability of CB [n] . Recent synthesis of perhydroxyCB $[n]$ via direct functionalization, ${ }^{7}$ enabled us to devise and synthesize a wide variety of $\mathrm{CB}[n]$ derivatives, ${ }^{8,4}$ which opened up opportunities to explore many applications of $\mathrm{CB}[n]$ including their use in separation science. A general approach and preliminary results on the production of $\mathrm{CB}[n]$-based stationary phase materials and their uses in chromatography have been disclosed in patent literature. ${ }^{10,11}$ Recently, Liu $e t$ al. ${ }^{12}$ reported a perhydroxyCB $[6]$ bonded silica gel material and its use in HPLC as a stationary phase to separate alkaloids. Despite successful separation of a mixture of alkaloids, it seemed to suffer from band broadening; the bandwidths of the peaks were rather wide. It could be due to the anomalous behavior of residual silanol groups on the silica surface since they did not carry out an end-capping reaction to block the silanol groups presumably because of the residual hydroxy groups on the $\mathrm{CB}$ moieties. These polar hydroxy groups would enable applications of the stationary phase for hydrophilic chromatography ${ }^{12}$; however, they would also hamper an end-capping reaction.

A group of us working at POSTECH recently reported the synthesis and characterization of a cucurbituril anchored silica gel via reaction of perallyloxyCB[6] and mercaptopropyl functionalized silica gel. ${ }^{13}$ Unfortunately, however, the separation efficiency of the stationary phase was rather disappointing. It may be due to the fact that the $C B$ molecules were attached only to the outer surface but not to the inner pores of the silica particles since UV irradiation, which could not effectively penetrate into the inner pores, was used to activate chemical bonding of the $\mathrm{CB}$ derivatives to the silica surface. In this study, cross polymerization was used to enable uniform attachment of the $\mathrm{CB}$ derivative to both outer surface and inner pores of silica particles. The approach of cross polymerization between silica gel and ligands, which has been used in the synthesis of chiral stationary phases with macromolecular ligands such as cellulose derivatives, ${ }^{1+20}$ is also found usefill for the synthesis of a CB anchored stationary phase in this study. The adoption of cross polymerization in this study resulted in additional merits. This $\mathrm{CB}$ derivative does not have any hydroxyl group, thus end-capping for the residual silanol groups of silica can be done without anomalous side reactions. In addition, further modification of the phase is possible owing to residual double bonds.

The other group of us at Inha Lniversity has been specialized in preparation of microcolumns $\mathrm{s}^{21-23}$ and joined this study for the examination of chromatographic performances. Incorporation of microcolumn technology to this study was beneficial in that the needed amount of $\mathrm{CB}$ derivatives for preparation of a new stationary phase and its chromato- 
graphic applications was only a few hundred milligrams. which enabled dramatic saving in time and labor. Herein we report a new $\mathrm{CB}[6]$-based stationary phase made by cross polymerization and by double end-capping, which shows good separation efficiencies for nonpolar and polar solutes. In particular, the double end-capping has been proven to improve the separation efficiency of the $\mathrm{CB}[6]$-based stationary phase materials.

\section{Experimental}

Chemicals and apparatus. Methanol, tetrahydrofuran and water were of HPLC grade and purchased from Fisher (Pittsburg, PA, USA) and used without purification. Allyltrimethoxysilane, 1,1,1,3,3,3-hexamethyldisilazane (HMDS). trimethylchlorosilane (TMCS), trifluoroacetic acid (TFA), phenol, acetophenone, benzene, toluene, ethylbenzene. propylbenzene, butylbenzene, amylbenzene, 2,2-azobisisobutyronitrile (AIBN), ortho- and para-phenylenediamines. ortho-, meta-, and para-nitroanilines, ortho- and paraxylenes were purchased from Aldrich (Milwakee, IL, USA). Silica gel (Lichrosorb, $5 \mathrm{~mm}, 100 \AA$ ) was purchased form Merck (Whitehouse Station, NJ. USA). PerallyloxyCB[6] was prepared according to our reported procedure. ${ }^{7} \mathrm{~A}$ commercial C18 phase (Alltima C18, $5 \mu \mathrm{m}, 100 \AA$ ) was obtained from Alttech (Deerfield, IL. USA).

Infrared spectra were recorded on a Perkin Elmer Spectrum GX, FT- $\mathbb{R}$ spectrometer using $\mathrm{KBr}$ as media. The ${ }^{l} \mathrm{H}$ XMR spectra and ${ }^{13} \mathrm{C}$ NMR spectra were recorded on a Bruker DRX500 spectrometer. A Shimadzu (Tokyo, Japan) 10AD pump. a Shimadzu DGL-14A membrane degasser, a Rheodyne (Cotati. CA. USA) 7520 injector with a $0.5 \mu \mathrm{L}$ injection loop, an Isco (Lincoln, NE, USA) CV4 capillary window detector, and the home-made $0.5 \mathrm{~mm}$ ID glass-lined microcolumn were combined to compose the HPLC system. The chromatographic data were obtained by a PC system. and the software Multichro 2000 from Youlin-Gisul (Sungnam, Korea) was used to acquire and process the data. An Alltech (Deerfield, IL,USA) slurry packer was used to pack the microcolumns used.

Preparation of allylsilica. HPLC grade porous silica gel (Lichorosorb, $5 \mu \mathrm{m}, 100 \AA$ ) was baked at $300^{\circ} \mathrm{C}$ for $3 \mathrm{~h}$ to remove adsorbed water completely. It ( $1 \mathrm{~g}$ ) was dispersed in toluene and reacted with allyltrimethoxysilane $(0.7 \mathrm{~mL})$ for $3.5 \mathrm{~h}$ at $110^{\circ} \mathrm{C}$ with stirring. Then. HMDS $(0.4 \mathrm{~mL})$ was added for end-capping of residual silanol groups and the reaction mixture was stirred for $1 \mathrm{~h}$ at the same temperature. After completion of the reaction, the allylsilica 1 was filtered, thoroughly washed with toluene, THF, 50/50 (v/v $\%$ ) methanol/water, and methanol, and dried. FT-IR ( $\mathrm{KBr}$ ) $\left(\mathrm{cm}^{-1}\right.$ ): 1642 (allyl), 1095 (Si-O-Si), 914 (Si-O). Elemental analysis: $\mathrm{C}, 5.2 ; \mathrm{H}, 1.1 \%$. The elemental analysis data indicate that $0.70 \mathrm{mmol}$ of allylsilane was tethered per gram of the silica gel.

Preparation of the stationary phase via cross polymerization. Allylsilica 1 (600 mg), perallyloxyCB[6] 2 (120 $\mathrm{mg})$, and chloroform $(15 \mathrm{~mL})$ were added to a small round- bottomed flask and stirred well and evaporated to dryness in a rotavapor. A solution containing anisole $(0.3 \mathrm{~mL})$ and AIBN (24 mg) dissolved in toluene $(15 \mathrm{~mL}$ ) was added to the flask, stirred, and evaporated. The addition of anisole is a critical factor in our process. Anisole is not evaporated by a rotavapor, thus it soaks the entire silica surface and functions as a solvent for the monomers. The bath temperature was raised to $110^{\circ} \mathrm{C}$ and maintained for $24 \mathrm{~h}$ while the reaction flask was rotated. The stationary phase 3 was then thoroughly washed with chloroform and methanol in sequence and dried. FT-IR ( $\mathrm{KBr})\left(\mathrm{cm}^{-1}\right)$ : 1761 (CB[6], carbonyl), 1646 (unreacted allyl groups of $\mathrm{CB}[6]$ ), 1113 (Si-O-Si), 937 (SiO) (Figure 1). Elemental analysis: $\mathrm{N}, 1.4 ; \mathrm{C}, 9.2 ; \mathrm{H}, 1.5 \%$. Elemental analysis shows that $45 \mu \mathrm{mol}$ of $\mathrm{CB}[6]$ was attached per gram of the silica support.

A portion of the phase 3 was packed in a microcolumn $(0.5 \mathrm{~mm} \times 300 \mathrm{~mm})$ for investigation of chromatographic performance. The rest of the phase $\mathbf{3}$ was divided into two groups for secondary end-capping. Only HMDS was used for one group while a 3:1 mixture of HMDS and TMCS was used for the other group. Thus in the case of the first group, the stationary phase $3(100 \mathrm{mg})$, HMDS $(40 \mu \mathrm{L})$, and toluene $(2 \mathrm{~mL})$ were added to a small round bottomed flask and stirred at $110{ }^{\circ} \mathrm{C}$ for $4 \mathrm{~h}$ under reflux. A mixture of HMDS $(30 \mu \mathrm{L})$ and TMCS $(10 \mu \mathrm{L})$ was used instead of HMDS $(40 \mu \mathrm{L})$ for the second group. The final stationary phases were thoroughly washed with toluene and methanol in sequence and dried. Each of the stationary phases was packed in a microcolumn $(0.5 \mathrm{~mm} \times 300 \mathrm{~mm})$ for study of chromatographic performance according to the procedure of previous studies. ${ }^{21,23}$

\section{Results and Discussion}

Preparation and characterization of allylsilica. Allylsilica was prepared according to the reported procedure ${ }^{16}$ with minor modifications (Scheme 1). The allylsilica was then end-capped using HMDS to give the end-capped allylsilica 1, which was characterized by FT-IR and elemental analysis. The elemental analysis (based on carbon content) showed attachment of $0.70 \mathrm{mmol}$ of allylsilane per gram of silica gel.

Preparation and characterization of perallyloxyCB[6] anchored phases. $C B$ derivative 2 was prepared by the literature procedure [7]. Cross polymerization was employed

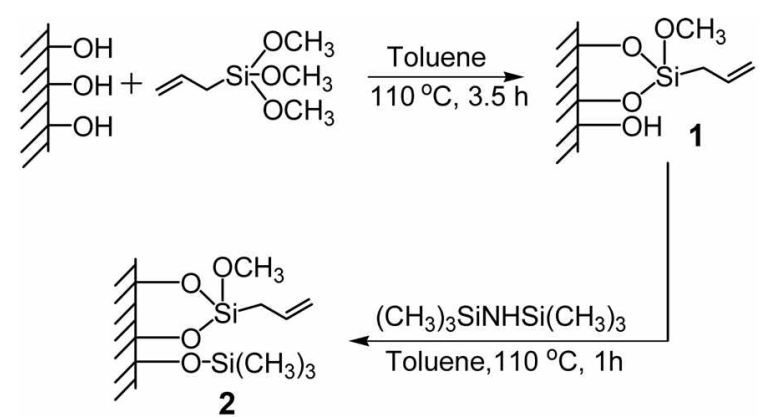

Scheme 1. Preparation of allylsilica 1. 


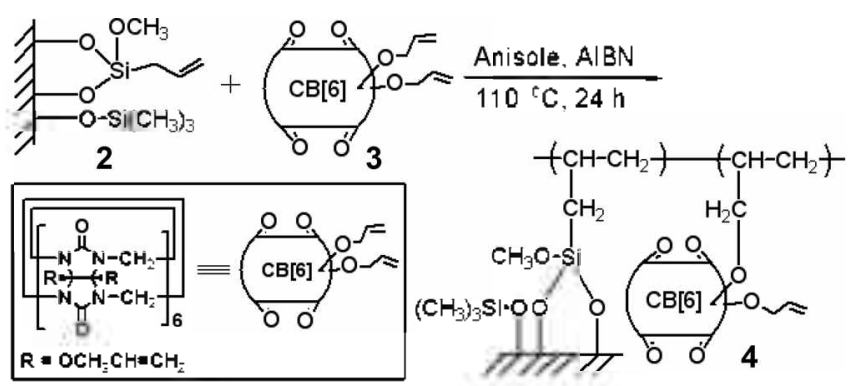

Scheme 2. Preparation of perallyloxyCB[6] 2 anchored silica phase through cross polymerization.

to couple end-capped allylsilica 1 and perallyloxyCB[6] 2 to prepare the perallyloxyCB $[6]$ anchored stationary phase $\mathbf{3}$ (Scheme 2). The allylsilica 1 was first physically coated with 2 and then the cross polymerization reaction was carried out. The FT-IR spectrum of 3 (Figure 1) showed absorption bands at $1761 \mathrm{~cm}^{-1}$ and $1646 \mathrm{~cm}^{-1}$ attributed to the carbonyl and unreacted allyl groups of compound 2 .

Chromatographic performance of the new stationary phases. Separation performances of the stationary phases prepared with single end-capping, and double end-capping (the first and second groups) are shown in Figure 2. A mixture of alkylbenzenes was used as a test mix. Thorough end-capping was found to be a very critical factor in the $\mathrm{CB}$ anchored phase. The examination of Figure 2 reveals that the separation efficiency and quality of the phases prepared with double end-capping are much better than that with single end-capping. In case of double end-capping. the second group (a mixture of HMDS and TMCS was used) showed a better performance ( 15000 vs 7400 as $\mathrm{N}$ of propylbenzene. for example) than the first group (only HMDS was used). The peaks of the phase of single end-capping (Figure 2A) were accompanied with severe tailing and wide bandwidth. and they were not well separated in $60 / 40(\mathrm{v} / \mathrm{v} \%)$ methanol $/$ water $(0.1 \% \mathrm{TFA})$, thus an eluent of $40 / 60(\mathrm{v} / \mathrm{v} \%)$ methanol $/$ water $(0.1 \%$ TFA) was used to get separated peaks. Bandwidths and tailing were dramatically reduced when the phase was doubly end-capped (Figure $2 \mathrm{~A}$ and $2 \mathrm{~B}$ ). The

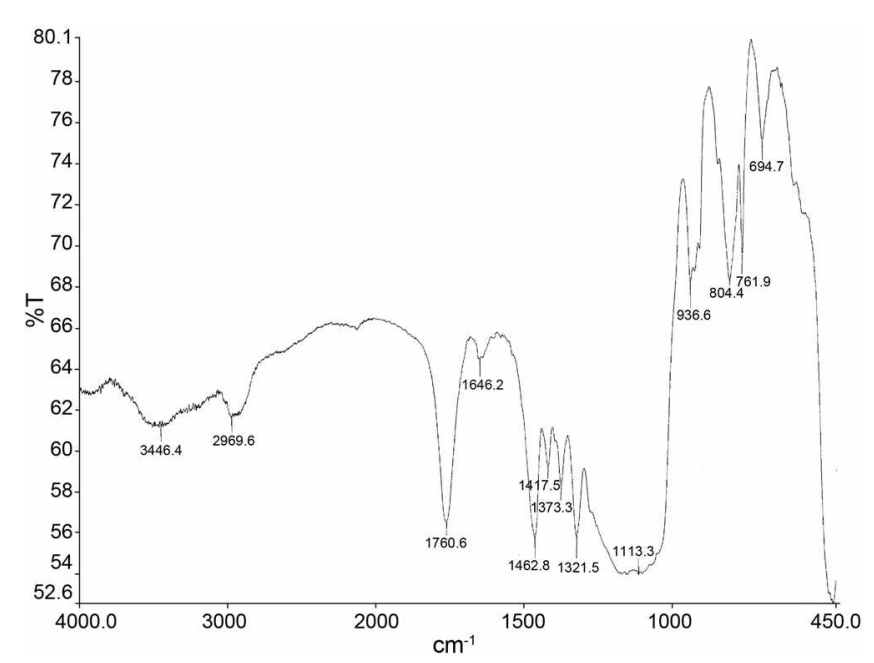

Figure 1. FT-IR spectrum of perallyloxyCB[6] anchored silica gel.
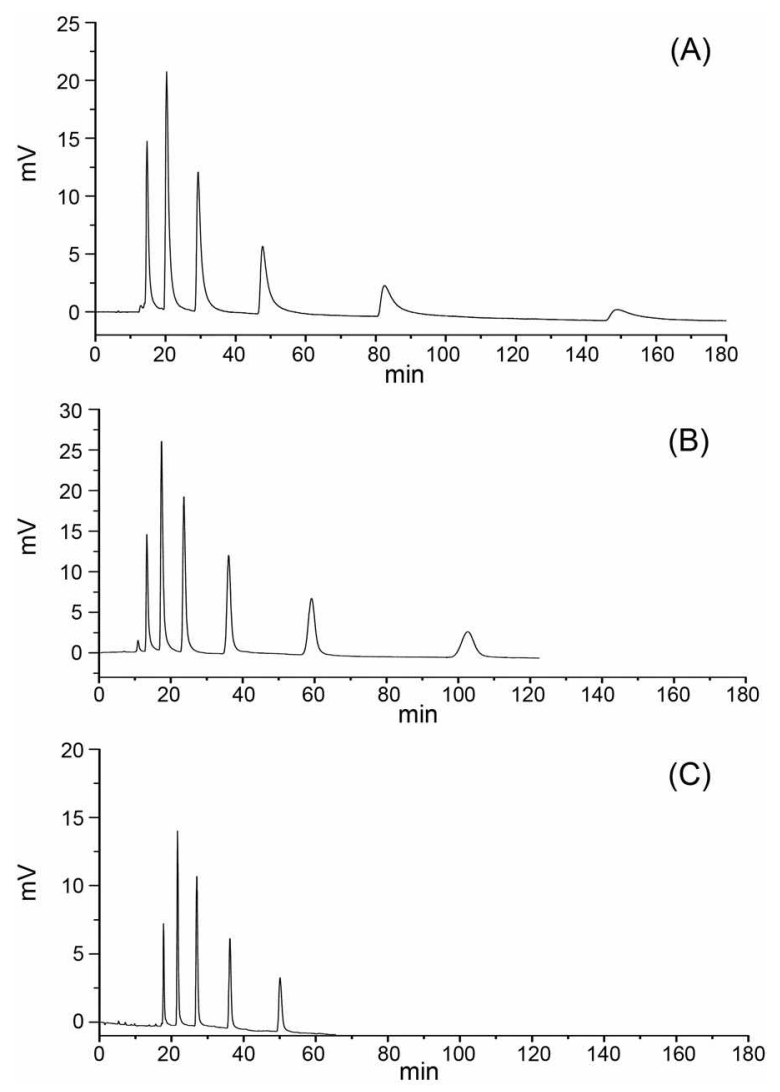

Figure 2. The chromatogram of alkylbenzenes obtained by the column packed with (A) the phase 3 of single end-capping eluted with $40 / 60(\mathrm{~V} / \mathrm{\gamma} \%)$ methanol water with $0.1 \%$ TFA. (B) the first group stationary phase (HMDS only) of double end-capping eluted with $60 / 40(v / \% \%)$ methanol/water with $0.1 \%$ TFA, and $(C)$ the second group stationary phase (HMDS:TMCS mixture) of double end-capping eluted with $60.40(\mathrm{v} / \mathrm{v} \%)$ methanolwater with $0.1 \%$ TFA. Elution order: benzene, toluene, ethylbenzene, propylbenzene, butylbenzene, amylbenzene.

superiority of the second group of the doubly end-capped phases over the first group also seems to be related to better quality of end-capping. The use of both HMDS and TMCS secured complete end-capping while the use of only HMDS or TMCS did not. As the molecular size of TMCS is smaller than that of HMDS, it can penetrate freely into small pores of silica to end-cap even hidden silanol groups deep inside the silica structure. However, using only TMCS is not good since TMCS generates $\mathrm{HCl}$ when it reacts with a silanol group. Since HMDS is a base, it not only end-caps silanol groups in open space but also neutralizes $\mathrm{HCl}$ generated from the reaction of TMCS with silanol groups. As our preparation scheme of stationary phases was well compatible with double end-capping, the resulting stationary phase showed well separated and nice looking peaks. Our stationary phase was also good for separation of polar compounds. The chromatogram of a mixture of polar compounds (phenol, 2-nitroaniline, acetophenone) and nonpolar compounds (benzene and toluene) is shown in Figure $3 \mathrm{~A}$. Thus, our phase has shown good separation efficiencies for nonpolar and polar compounds as a benefit of complete endcapping. 

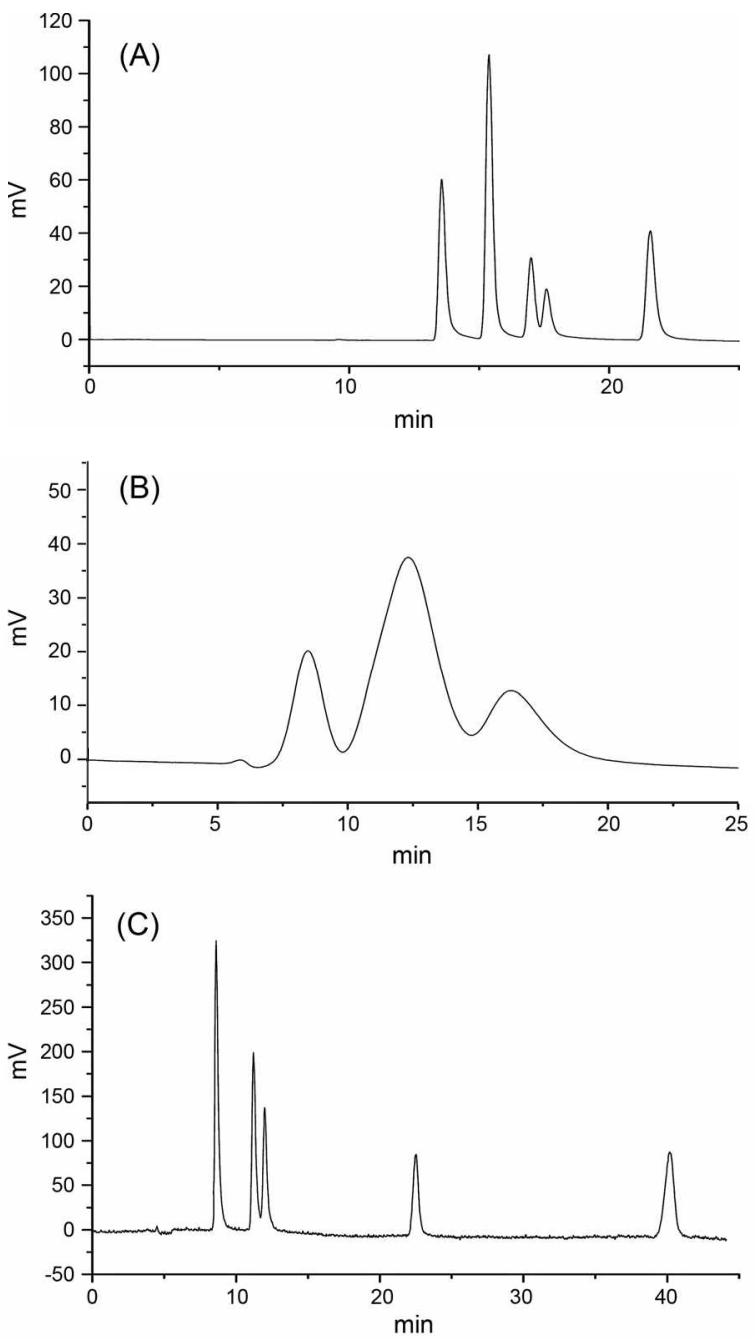

Figure 3. The chromatogram of test mix 2 obtained by A) the column packed with the second group stationary phase (HMDS: TMCS mixture) of double end-capping, B) the column packed with end-capped allylsilica, and C) the column packed with the commercial Alltima $C 18$ phase. The relative composition of ingredients of the sample used in C) was different from that used in A) and B) since a new batch of test mix was prepared when the C) work was performed. Mobile phase: $60 / 40(\mathrm{v} / \mathrm{V} \%)$ methanol/water with $0.1 \%$ TFA. Elution order: phenol, 2-nitroaniline, acetophenone, benzene. toluene.

Critical examination of separation performances of the doubly end-capped cucurbituril phase in comparison with the end-capped allylsilica and a commercial $C 18$ phase. A column packed with end-capped allylsilica was prepared to check if end-capped allylsilica alone can give some separation performance. As shown in Figure 3B, endcapped allylsilica was found unable to give good separation efficiency.

A column packed with a commercial C18 phase (Allima $\mathrm{C} 18,5 \mu \mathrm{m}, 100 \AA$, carbon load $16 \%$ ) was also prepared for critical comparison in separation efficiencies.

It was difficult to find definite evidences of our perallyloxyCB anchored phase for better separation performances in comparison with the widespread and versatile commercial $\mathrm{C} 18$ phases. There is an advantage of the commercial C18 phase over our phase. A single alky! ligand is introduced to each silanol site in the $\mathrm{C} 18$ phase while an unknown number of $\mathrm{CB}$ units are introduced to each silanol site since each $\mathrm{CB}$ unit has many doubles bonds. In other words, the $\mathrm{C} 18$ phase is rather homogeneous while our $\mathrm{CB}$ anchored phase is heterogeneous. A perallyloxyCB $[6]$ anchored stationary phase with a higher $\mathrm{CB}$ content (carbon load -20\%) was prepared using a larger amount of the $\mathrm{CB}$ derivative 2 with a prolonged reaction time ( $72 \mathrm{~h}$ instead of $24 \mathrm{~h}$ ). In this case, however, the size and shape of the resulting $\mathrm{CB}$ attached silica were irregular and its separation efficiency was not as good as that of the phase 3 , which may be due to the formation of polymer particles of 2 on the outer surface of the silica.

As mentioned above, the carbon load of our phase was forced to be limited to a low value (less than $10 \%$ ) due to its heterogeneity and solid-like character while the $\mathrm{C} 18$ phase was capable of a higher carbon load $(16 \%)$ with liquid-like character. Naturally, the $\mathrm{C} 18$ phase showed better separation performances in general.

As shown Figure 3C, the separation efficiency of the Alltima $\mathrm{C} 18$ phase was better than that of our phase (Figure $3 \mathrm{~A}$ ). It should be noted that the results of Figure $3 \mathrm{~A}$ and $3 \mathrm{C}$. proved that the carbon load of the Alltima C 18 phase is higher than that of our phase and that the polarity of our phase is much higher than that of the Alltima C18 phase owing to cucurbituril moities. Retention times of nonpolar solutes (benzene and toluene) in the Alltima C 18 phase were much longer than those in our phase owing to the higher carbon load of the Alltima C 18 phase while retention times of polar solutes (phenol, 2-nitroaniline, acetophenone) in our phase were longer than those in the Alltima C18 phase owing to the higher polarity of our phase in spite of the lower carbon load.

The advantage of the CB phase developed in this study over the commercial $\mathrm{C} 18$ phase resides in the selective usage of host-chemistry property exhibited from the $\mathrm{CB}$ phase. There are, however, some limitations in this approach. Too strong host-guest interaction caused too slow mass transfer kinetics and consequent band broadening and/or tailing. Too loose host-guest interaction, on the other hand, did not give enough separation selectivity. The host-guest interaction was dependent on the polarity, size and shape of guest molecule, and $\mathrm{pH}$ and composition of solvent (mobile phase), thus optimization was necessary if a pair of solutes of proper host-guest chemistry was chosen.

We managed to find a proper pair of solutes that meet the requirements. The optimized chromatographic resolution of ortho- and paraphenylenediamine by our phase (Figure 4B) was found better $(R=4.05)$ than the optimized separation ( $R$ $=3.18$ ) of the same pair of solutes by the Alltima C18 phase (Figure 4A). The clear evidence of incorporation of hostguest chemistry in their separation was also found in Figure $4 \mathrm{~B}$. In the C 18 phase, $p$-phenylenediamine elutes betore 0 phenylenediamine (Figure 4A) since o-phenylenediamine is less polar than $p$-phenylenediamine owing to formation of intramolecular hydrogen bond between the adjacent amino 

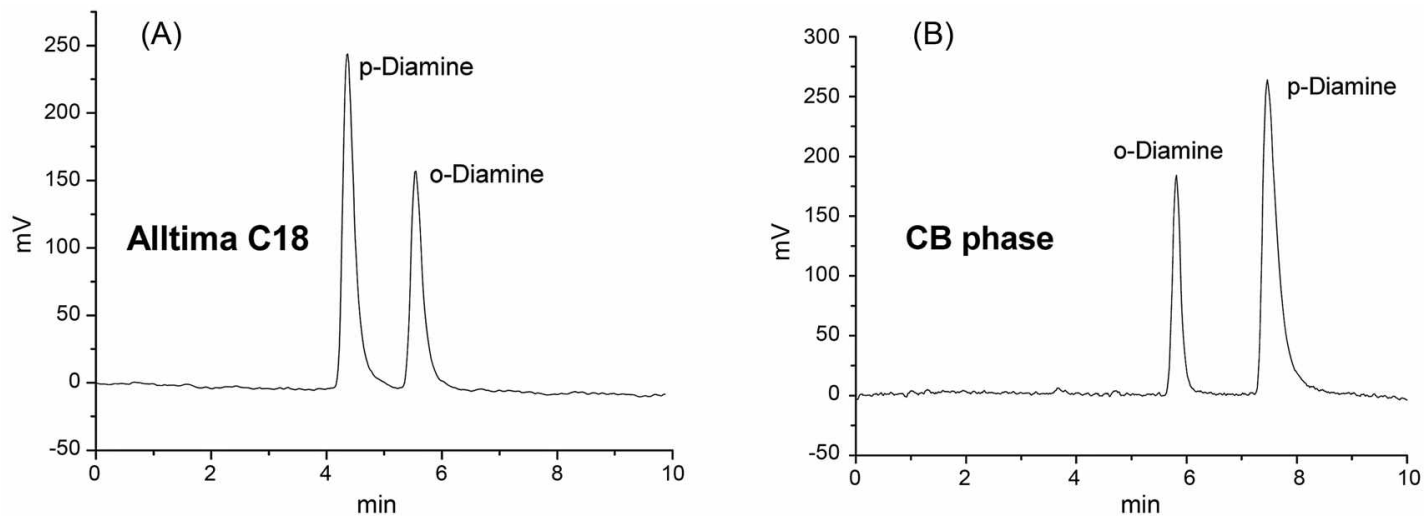

Figure 4. Comparison of optimized separation of ortho- and paraphenylenediamine between the Alltima C18 phase (A) and the cucurbituril phase (B). In (A). pH 4.5 (50 mM sodium actate/acetic acid) 60.40 methanoliwater was used as the eluent. while $\mathrm{pH} 7(50 \mathrm{mM}$ ammonium acetate) 60440 acetonitrile water was used as the eluent in (B). In both cases. a flow rate of $10 \mu \mathrm{L} / \mathrm{min}$ was used, and $254 \mathrm{~nm}$ was used as the detection wavelength.

groups. On the other hand, $o$-phenylenediamine elutes faster than $p$-phenylenediamine in our phase since $p$-phenylenediamine fits well to the cucurbituril cavity while $o$-phenylenediamine is rather excluded from the cavity.

A point that should be mentioned about the stationary phase of this study is its capability of further modification to yield new stationary phases with diverse properties by making use of the residual allyl groups on the $\mathrm{CB}$ unit. Various types of secondary ligands such as nonploar alkyl chains. polar moieties, ion exchange ligands, and chiral ligands, etc. can be incorporated, which is subject to future study.

\section{Conclusion}

A CB [6]-anchored stationary phase has been prepared by cross polymerization and double end-capping. In general, this phase has shown good separation efficiencies for nonpolar and polar solutes. This phase showed better separation performance than the commercial $\mathrm{C} 18$ phase for the case where host-guest chemistry was properly incorporated in solute retention. This phase is expected to be susceptible to further modification of secondary ligands.

Acknowledgments. The Inha University part of this work was supported by grant $\lambda_{0}$. (R01-2006-000-10004-0) from the Basic Research Program of the Korea Science \& Engineering Foundation and the BK 21 Program (Korea Ministry of Education). We also acknowledge the Creative Research Initiative Program (Korean Ministry of Science and Technology) and the BK 21 Program (Korean Ministry of Education) for support of the POSTECH part of this work.

\section{References}

1. Mock, W. L. Top. Cum Chent. 1995, 175, 1-24.
2. Kim, K. Chem. Soc. Rev. 2002, 31, 96-107.

3. Kim, J.; Jung, 1.-S.; Kim, S.-Y; Lee, E.; Kang, J.-K.; Sakamoto, S.; Yanaguchi, K.; Kim, K. J.Am. Chem. Soc. 2000, 122, 540-541.

4. Liu, S.; Ruspic, C.; Mukhopadhyay, P.; Chakrabatri, S.; Zavalij, P. Y.; Isaacs, L. J. Am. Chem. Soc. 2005, 127, 15959-15967.

5. Lee, J. W.; Samal, S.; Selvapalam, N.; Kim, H. J.; Kim, K. Acc. Chem. Res. 2003, 36,621-630.

6. Lagona, J.; Mukhopadlyay, P.; Chakrabarti, S.; lsaacs, L. Angen: Chem., Int. Ed. 2005, 44, 4844-4870.

7. Jon, S. Y.; Selvapalam, N.; Oh, D. H.; Kang, J.-K.; Kim, S.-Y.; Jeon, Y. J.; Lee, J. W. Kim, K. J. An. Chem. Soc. 2003, 125, 10186-10187.

8. Lee, H.-K.; Park, K. M.; Jeon, Y. J.; Kim, D.; Oh, D. H.; Kim, H S.; Park, C. K ; Kim, K. J. Am. Chem. Soc. 2005, /27, 5006-5007.

9. Jeon, Y. J.; Kim, H.; Jon, S.; Selvapalam, N.; Oh, D. H.; Seo, I.; Park, C.-S.; Jung, S. R.; Koh, D.-S.; Kim, K. J. Am. Chem. Soc. 2004, 126, 15944-15945.

10. Kim, K.; Balaji, R.; Oh, D. H.; Ko, Y. H.; Jon, S. Y. PCT WO 2004:072151 Al, 2004, KR 0008453, 2003.

11. Kim, K.; Oh, D. H.; Nagarajan, E. R.; Ko, Y. H.; Samal, S. PCT WO 2005/010058 Al, 2005, KR 0051840, 2003.

12. Liu, S. M.; Xu, L.; Wu, C. T.; Feng, Y. Q. Talanta 2004, 64, 929934

13. Nagarajan, E. R.; Oh, D. H.; Selvapalam, N.; Ko, Y. H.; Park, K M. Kim, K. Tetrahedron Lett. 2006, 47, 2073-2075.

14. Minguillon, C.; Franco, P; Oliveros, L.; Lopez, P. I. Chromatogr: 4 1996, $728,407-414$.

15. Minguillon, C.; Franco, P.; Oliveros, L. J. Chrontatogr: A 1996, $728,415-422$.

16. Oliveros, L.; Senso, A.; Minguillon, C. Chrrality 1997, 9, 145-149.

17. Kubota, T.; Yanamoto, C.; Okamoto, Y. Chirality 2003, 15, 77-82.

18. Kubota, T.; Yamamoto, C.: Okanoto, Y. J. Polym Sci., Part A: Polvm. Chem. 2004, 42, 4704-4710.

19. Kubota, T.; Yamamoto, C.; Okamoto, Y. I. Polmm Sci. Part A: Polm Chem. 2003, 41,3703-3712.

20. Chen, X.; Qin, F.; Liu, Y.; Huang, X.; Zou, H. $J$. Chromatogr: $A$ 2004, $1034,109-116$.

21. Cheong, W. J.; Seo, Y. J.; Park, S. T.; Kang, G. W. Bull. Korean Chem. Soc. 2006, 27, 1059-1062.

22. Seo, Y. J.; Kang, G. W.; Park, S. T.; Moon, M.; Park, J. H.; Cheong, W. I. Bull. Korean Chem. Soc. 2007, 28, 999-1004.

23. Cheong, W. T. J. Chromatogr: A 2005, 1066, 231-237. 